\title{
La firma electrónica avanzada notarial y sus retos en Uruguay*
}

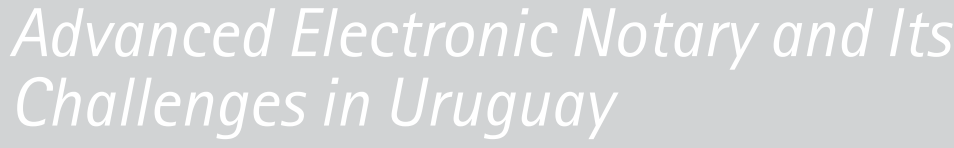

\section{Elisabeth Bouvier Villa**}

\begin{abstract}
RESUMEN
Hoyvivimosen una "Sociedad Red", donde la información y la comunicación asi como el enorme almacenamiento y tráfico de datos, han impactado en todos los ámbitos sociales, y el notario no es ajeno a esto en el ejercicio de su función notarial. Las herramientas tecnológicas, en especial el uso de Internet, las redes sociales y la firma electrónica, han producido una revolución sin precedentes dentro del notariado global y, en forma particular, dentro del notariado uruguayo por sus propias caracteristicas. En este trabajo, comenzaremos por hacer un breve análisis de la legislación uruguaya en materia de firma electrónica y firma electrónica avanzada notarial. Luego, visualizaremos cuáles son los retos que el Notariado uruguayo tiene frente a la irrupción de estas herramientas tecnológicas y al gran desarrollo del comercio electrónico. Retos que tendrá que gestionar sin afectar en nada la esencia de la función notarial y de la fe pública, elementos esenciales de la profesión.
\end{abstract}

\section{PALABRAS CLAVE}

Notariado, Uruguay, firma electrónica, función notarial, legislación.

\begin{abstract}
Today we live in a "Network Society", where information and communication as well as the enormous storage and traffic of data, have impacted in all social areas, and the public notary is not an exception of this in the exercise of its function. The technological tools, especially the use of the Internet, social networks and electronic signature, have produced an unprecedented revolution within the global public notary and, in particular, within the Uruguayan notary by its own characteristics. In this paper, we will begin by making a brief analysis of Uruguayan legislation in the field of electronic signatures and advanced electronic notary signature. Then we will visualize the challenges that the Uruguayan Notary has had to face, sudden appearance of these technological tools and the great development of electronic commerce. Challenges that will have to manage without affecting in any way the essence of the notary function and the public faith, essential elements of the profession.
\end{abstract}

\section{KEYWORDS}

Notary, Uruguay, electronic signature, notarial function. Legislation.

\footnotetext{
*Artículo recibido el 8 de junio de 2017 y aceptado el 21 de septiembre de 2017

**Asociación de Escribanos del Uruguay, Comisión de Derecho Informático y Tecnológico. (escbouvier@gmail.com) orcid 0000-0002-4001-711X
} 
SUM ARIO

1. Introducción

2. Uruguay: legislación en materia de firma electrónica

3. Retos del notariado uruguayo frente al uso de la firma electrónica avanzada notarial

4. Conclusiones

\section{Introducción}

Las nuevas tecnologías enfrentan a la profesión notarial con nuevos paradigmas y grandes desafíos en el ejercicio de su función. Esto significa un gran cambio cultural para el notariado, quien desde miles de años está asociado con el papel. El notariado debe involucrarse hoy en estos nuevos paradigmas. Siempre debe tener presente que la función notarial no cambia en su esencia; lo único que cambia es su técnica. Esta realidad ha impactado fuertemente al notariado uruguayo, sobre todo por tener determinadas características que le hacen aún más difícil gestionar este cambio.

La más importante es que actuamos en ejercicio libre de la profesión, a diferencia del resto de los notarios que integran el notariado latino. ¿Qué significa esto? Que cualquier notario uruguayo puede ejercer su profesión en todo el territorio nacional libremente. Esta característica se ve agravada cuando nos enfrentamos a un escenario con 7015 notarios activos (al 31 de diciembre 2016) ${ }^{1}$ para una población de 3440157 personas (al 30 de junio 2013). ${ }^{2}$

A pesar de todas estas dificultades, el notariado uruguayo tiene una actitud proactiva, a los efectos de incorporar las herramientas tecnológicas y, en especial, la firma electrónica avanzada notarial en el ejercicio de su función. La gran dificultad para el notario, al momento del cambio de paradigma, es conciliar la seguridad jurídica con la seguridad tecnológica de las herramientas.

Esta conciliación es primordial al momento de generar confianza en el uso de la firma electrónica avanzada. Por ello, fue el objetivo principal al momento de reglamentar su uso en la función notarial. La solución a la que se arribó fue la creación del soporte notarial electrónico anexado a la firma electrónica

\footnotetext{
1 "Anexos a las notas a los estados contables. Nota 11". Caja Notarial. [Consulta: 15 de septiembre, 2017]. Disponible en: http://www.cajanotarial.org.uy/innovaportal/file/143/14/anexos-ec-2016.pdf

2 "Uruguay en cifras 2014", Instituto Nacional de Estadistica. [Consulta: 15 de setiembre, 2017]. Disponible en: http://www.ine.gub.uy/documents/10181/39317/Uruguay_en_cifras_2014.pdf/aac28208-4670-4e96-b8c1-b2abb$93 b 5 b 13$
} 
avanzada notarial. Por tratarse de una solución muy particular dentro del notariado latino, consideramos importante investigar sobre este tema.

De la investigación realizada, surge que el notariado uruguayo no ha logrado gestionar este cambio de paradigma. Si bien la ley 18 600, "Documento electrónico y Firma electrónica. Se reconocen su validez y eficacia jurídica”, fue aprobada en el año 2009, es recién el 4 de febrero de 2015 que la Suprema Corte de Justicia (órgano que ejerce la superintendencia del notariado uruguayo) aprobó la Acordada 7831 que permite el uso de la firma electrónica avanzada notarial en la función notarial. Hoy, a más de dos años de dicha aprobación, el notariado no ha incorporado al ejercicio de su función esta herramienta tecnológica. El cambio de mentalidad en el gremio notarial es fundamental si pretendemos dar respuesta a los ciudadanos de este siglo XXI.

El cambio, en la técnica notarial, deberá gestionarse a sabiendas de que estamos ante una herramienta tecnológica segura y confiable por sus características técnicas. Luego de las investigaciones sobre los avances tecnológicos que se avizoran, podemos afirmar que ellos no podrán sustituir al notario persona en principios fundamentales e irrenunciables de la función notarial (como el control de legalidad y el consentimiento informado) al momento de otorgar actos y contratos en los que actúa el notario. Los mismos son pilares de primera línea para la seguridad jurídica que tanto necesita la sociedad para desarrollarse con equidad y paz social.

\section{Uruguay: legislación en materia de firma electrónica}

Si bien en Uruguay existían leyes y decretos referidos a la firma electrónica en el ámbito del Estado, desde el año 2009 se legisló en forma específica sobre la materia. Así, se aprobó la Ley 18600 de 21 de setiembre de 2009 y su Decreto Reglamentario 436/2011 de fecha 8 de diciembre de 2011. Existen modificaciones a la misma en las leyes 18996 de 7 de noviembre de 2012, artículos 41 y $42^{3}$ y 19 355, de 30 de diciembre de 2015, artículo 85. ${ }^{4}$ A continuación, haremos un análisis de la ley en aquellos aspectos que son de interés para el tema objeto de este trabajo.

\footnotetext{
3 "Ley 18996 del 7 de noviembre de 2012", Centro de Información Oficial Impo. [Consulta: 5 de febrero, 2017]. Disponible en: http://www.impo.com.uy/bases/leyes-originales/18996-2012

4 "Ley 19355 del 30 de diciembre de 2015", Centro de Información Oficial Impo. [Consulta: 5 de febrero, 2017]. Disponible en: http://www.impo.com.uy/bases/leyes-originales/19355-2015
} 
Queremos destacar que la ley en estudio no altera ni limita la función notarial ya que en su artículo 1 expresa:

Queda reconocida la admisibilidad, validez y eficacia jurídicas del documento electrónico y de la firma electrónica. Los servicios de certificación deberán ajustarse a lo previsto en esta ley, su actividad no estará sujeta a autorización previa y se realizará en régimen de libre competencia, sin que ello implique sustituir o modificar las normas que regulan las funciones que corresponde realizar a quienes están facultados legalmente para dar fe pública. Las disposiciones de esta ley no alteran el Derecho preexistente respecto a la celebración, perfeccionamiento, validez y eficacia de los actos y negocios jurídicos (las cursivas son nuestras).

La ley, en su artículo segundo, realiza una serie de definiciones, técnica que consideramos apropiada al momento de aplicación de la misma ya que estamos en un campo de estudio donde se utilizan conceptos hasta ahora desconocidos para el derecho.

La ley uruguaya distingue entre firma electrónica y firma electrónica avanzada, y les otorga diferentes efectos jurídicos. Las define en su artículo 2 incisos J y K. En el numeral 5 del inciso K, al definir la firma electrónica avanzada encontramos el concepto de certificado reconocido. Es algo que queremos destacar, ya que la ley distingue entre certificado electrónico, artículo 2 inciso $\mathrm{B}$, y certificado reconocido, en el mismo artículo inciso C. ${ }^{5}$ El certificado reconocido es el expedido por el prestador de servicio de certificación acreditado ante la unidad de certificación electrónica.

Esta unidad creada por la ley es ante quien se acreditan los prestadores de servicios de certificación. Es la encargada de implementar las políticas para la mejor aplicación de la normativa, entre otras tareas. Resaltamos al prestador de servicio de certificación acreditado, porque de acuerdo con lo establecido en la ley es el único que puede expedir la firma electrónica avanzada. Los artículos 5 y 6 establecen los efectos jurídicos de la firma electrónica y la electrónica avanzada respectivamente. Esta última es aquella a la que se le otorga, según el artículo 6 referido, "idéntica validez y eficacia que la firma autógrafa consignada en documento público o en documento privado con firmas certificadas,

\footnotetext{
5 "Ley 18600 del 21 de setiembre de 2009", Centro de Información Oficial Impo. [Consulta: 15 de diciembre, 2016]. Disponible en: http://www.impo.com.uy/bases/leyes-originales/18600-2009
} 
siempre que esté debidamente autenticada por claves $u$ otros procedimientos seguros".

Dado el objeto del presente trabajo, no podemos dejar de subrayar el artículo 7, ya que es allí donde se autoriza el uso del documento electrónico y la firma electrónica avanzada en la función notarial. Este establece que dicho uso requerirá la reglamentación de la Suprema Corte de Justicia, organismo que ejerce la superintendencia del notariado en el Uruguay.

En el capítulo II de la ley se establece todo lo referente a la infraestructura nacional de certificación electrónica, en la que Uruguay siguió el modelo jerarquizado preceptivo para el Mercosur. En esa estructura de confianza, se crea la Autoridad Certificadora Raíz Nacional (artículo 15), primera en la cadena de confianza: la Agencia para el Desarrollo del Gobierno de Gestión Electrónica y la Sociedad de la Información y del Conocimiento (Agesic).

Luego, en esa cadena tenemos a los prestadores de servicios de certificación acreditados, a los cuales ya nos referimos. Actualmente, en Uruguay existen tres prestadores de servicios de certificación acreditados: el Correo, ente público; Abitab, empresa privada que actúa en régimen de libre competencia; y el Ministerio del Interior, que expide el documento de identidad electrónico desde el mes de mayo de 2015.

El referido documento cuenta con la firma electrónica avanzada del titular de la misma, aunque todavía no es obligatoria su obtención por parte de aquellos ciudadanos que tengan su cédula de identidad vigente. El empleo por parte de los ciudadanos del documento de identidad electrónico con firma electrónica avanzada supondrá un avance en la implementación del gobierno digital. Permitirá que el ciudadano firme documentos para realizar diferentes trámites ante el Estado.

La Suprema Corte de Justicia, en uso de las potestades encomendadas en el artículo 7 de la ley 18600, que ya referimos, dictó la Acordada 7831 de fecha 4 de febrero 2015. Buscaba reglamentar el uso de la firma electrónica avanzada en el ejercicio de la función notarial. Dicha acordada integra el Reglamento Notarial vigente a la fecha y aprobado por la Acordada 7533 de 22 de octubre de $2004 .{ }^{6}$ Se incorpora a dicho reglamento el título vII, "Uso de la firma electrónica avanzada notarial” (artículo 1 Acordada 7831). ${ }^{7}$ Consideramos de

\footnotetext{
6 "Acordada 7533 del 22 de octubre de 2004. Reglamento Notarial", Poder Judicial de la República Oriental del Uruguay. [Consulta: 15 de febrero, 2017]. Disponible en: http://www.poderjudicial.gub.uy/images/stories/circulares/2004/100ACORDADA7533REGLAMENTONOTARIAL-TEXTOCOMPLETO.PDF

7 "Acordada 7831 del 4 de febrero de 2015", Poder Judicial de la República Oriental del Uruguay. [Consulta: 15 de febrero, 2017]. Disponible en: http://www.poderjudicial.gub.uy/images/stories/circulares/2015/006-15_-_REF._ACOR-
} 
buena técnica haber incorporado esta reglamentación como un nuevo título al reglamento notarial ya que nos encontramos ante un tema con conceptos y características específicas por tratarse de la utilización de una herramienta tecnológica.

Este título VII se divide en tres capítulos que analizaremos brevemente.

En el capítulo primero se establece que "La única firma electrónica que podrá utilizar el Escribano Público en el ejercicio de su profesión será la que realice mediante el certificado reconocido emitido por un prestador de servicios de certificación acreditado" (artículo 291 incorporado al Reglamento Notarial). Es decir que el notario público sólo puede utilizar en el ejercicio de su función la firma electrónica avanzada. ¿Por qué se opta por la firma electrónica avanzada? Porque técnicamente es la firma electrónica más segura y además es la única a la que la ley le otorga idéntica validez y eficacia que a la firma autógrafa. El capítulo referido continúa estableciendo las responsabilidades, obligaciones y sanciones que tendrá el notario al momento de utilizar la referida firma.

El capítulo II regula todo lo referente al soporte notarial electrónico, que será administrado, generado y emitido por la Caja Notarial de Seguridad Social (artículos 297 a 302). La referida institución es quien hoy expide el papel notarial de actuación, único papel en el que el notario puede actuar. Por esta razón, será quien emita el soporte notarial electrónico, único soporte electrónico que puede utilizarse en la función notarial para expedir los documentos notariales electrónicos que la misma Acordada determina.

La Caja Notarial de Seguridad Social ${ }^{8}$ emite el soporte notarial solicitado, previa consulta en línea a la Suprema Corte de Justicia para constatar que el solicitante notario esté habilitado en el ejercicio de su función. "La solicitud de emisión del Soporte Notarial Electrónico y la firma del documento notarial respectivo deberán hacerse dentro del mismo día” (artículo 297). La utilización de la firma electrónica avanzada asegura al receptor la identidad de la persona que suscribe el documento, su integridad y el no repudio.

$\mathrm{Al}$ estar la misma contenida en el soporte notarial electrónico, se asegura 232 que quien suscribe el documento es un notario habilitado en el ejercicio de su función. Esta es la razón de la obligación impuesta por el citado artículo 297. La seguridad tecnológica que el notariado exigía se obtiene plenamente con la conjunción de estos dos elementos.

DADA_7831_-_Firma_electr\%C3\%B3nica_avanzada_notarial-1.pdf

8 "Solicitud de papel y soporte electrónico", Caja Notarial. [Consulta: 14 de abril, 2017]. Disponible en: http://www. cajanotarial.org.uy/innovaportal/v/2280/1/innova.front/solicitud-de-papel-y-soporte-electronico.html 
El capítulo III establece los documentos notariales electrónicos que el notario puede expedir, ya que esta reforma no habla ni de protocolo electrónico ni de registro de protocolizaciones electrónico. Es sólo un comienzo del ejercicio de la función notarial en el mundo electrónico. Por ello, el notario sólo puede expedir traslados notariales electrónicos definidos en el artículo 303 de la Acordada en estudio. Éstos se expedirán "con la sola finalidad de ser remitidos vía electrónica”, según el artículo 304, a órganos del Estado, Gobiernos departamentales o municipales, entes autónomos y servicios descentralizados o a escribanos públicos.

Dichos traslados "sólo serán válidos para la concreta finalidad para los que fueron solicitados”, según el artículo 305. También se pueden expedir certificados notariales electrónicos, los cuales tendrán el contenido establecido en los artículos 311 al 316. Resumiendo, el notario sólo puede expedir traslados notariales electrónicos y certificados notariales electrónicos en las condiciones que hemos analizado. Por esta razón, afirmamos que estamos ante una reforma muy tímida. Los traslados notariales electrónicos que se pueden expedir tienen que cumplir con los requisitos expresamente mencionados en la Acordada, los cuales están muy acotados en sus fines y receptores.

La misma no contempla los avances tecnológicos y su incorporación en el ejercicio de la función notarial en forma amplia. Estamos convencidos de que, en el corto plazo, habrá que realizar una nueva reforma para que el notariado uruguayo no quede al costado de la ruta, o sea desplazado en muchos nichos de trabajo que hoy no se visualizan con claridad.

\section{Retos del notariado uruguayo frente al uso de la firma electrónica avanzada notarial}

El notariado uruguayo tiene ante sí un escenario que le presenta nuevos paradigmas en el ejercicio de su función. El Estado uruguayo ha tenido un desarrollo sostenido del gobierno digital. Por tanto, el expediente electrónico, los trámites en línea y la firma electrónica avanzada notarial son una realidad que llegó para quedarse.

Ante estos nuevos paradigmas, el notario tiene que gestionar un cambio cultural sin precedentes. Debe asumir este reto con responsabilidad y firmeza, teniendo en cuenta que la función notarial en su esencia no cambia, pero sí cambia la técnica. Estos nuevos paradigmas a los que nos enfrentamos nos presentan herramientas tecnológicas que hacen el ejercicio de nuestra función más eficiente, y nos permiten estar más cerca del ciudadano. 
Tal ciudadano necesita hoy más que nunca el asesoramiento del notario. Las relaciones jurídicas de las partes en el comercio electrónico y en el mundo digital en general no son entre pares como las del Código Civil. Estamos ante relaciones muy asimétricas, donde prima la voluntad del más fuerte: grandes empresas constructoras, tecnológicas, frente a las cuales el "ciudadano común" es la parte débil de cualquier contratación. Frente a este escenario, ¿cuáles son los retos que el notario uruguayo tiene hoy?

Por decreto del Poder Ejecutivo 459/2016 de 30 de diciembre de 2016, se aprobó la Agenda Uruguay Digital, Transformación con equidad, 2020. En el objetivo vi se habla del Gobierno de cercanía, y expresamente se dice: "Impulsar una manera distinta de entablar la relación entre los ciudadanos y el Estado, promoviendo la transparencia, la rendición de cuentas, la participación ciudadana y el desarrollo de mejores servicios, generando canales de contacto directo con las personas y mejorando la calidad de atención en los servicios que se brindan". ${ }^{9}$

Este objetivo tiene como meta llegar al 2020 con 100\% de los trámites de la administración central "iniciados, seguidos y completados en línea”. Esto impacta sin duda en la actividad notarial, ya que la mayoría de esos trámites son realizados por el notario en el ejercicio de su profesión. La función notarial asesora, formativa, autenticante está presente en cada uno de esos actos.

El notario debe utilizar su firma electrónica avanzada notarial, así como también el ciudadano, su cliente. Para ello, el gremio en su totalidad debe gestionar este cambio sin precedentes en la historia del notariado nacional. El relacionamiento con el Estado cambia en forma sustancial, tanto desde el profesional como desde el ciudadano. La meta para el año 2016, determinada por el Estado, era alcanzar que 100\% de los trámites de la administración central iniciaran en línea.

$\mathrm{Al} 26$ de abril de 2017, 96\% de los mismos se inician en línea. ${ }^{10} \mathrm{Si}$ el gremio notarial no asume este cambio de mentalidad en el ejercicio de la función notarial, si no se involucra seriamente, corre el riesgo de que lo acusen de ser incapaz de gestionar los cambios necesarios para seguir la evolución tecnológica. El notario en ejercicio de su función público-privada debe ser un articulador entre el Estado y el ciudadano para lograr el involucramiento de estos últimos en los cambios de paradigmas.

\footnotetext{
9 "Agenda Uruguay Digital 2020: transformación con equidad", Uruguay Digital. [Consulta: 14 de abril, 2017]. Disponible en: http://uruguaydigital.gub.uy/wps/wcm/connect/urudigital/8f392a86-be63-4dcf-98d9-162e0933bb7f/ Agenda+Uruguay+Digital+2020+-+final+ES.PDF?MOD=AJPERESECContentCache=NONE

10 "Trámites en línea", Agesic. [Consulta: 5 de mayo, 2017]. Disponible en: https://www.agesic.gub.uy/innovaportal/v/6020/21/agesic/como-vamos.html?idPadre $=4588$
} 
Según Reglamento 1/ 2002 de 12 de marzo de 2002, la Dirección General de Registros posibilita la solicitud remota de los certificados registrales a través del sitio web del Registro de la Propiedad Mobiliaria e Inmobiliaria, Registro Nacional de Actos Personales y Registro de Personas Jurídicas, sección Registro Nacional de Comercio. El certificado debe retirarse en la oficina, formato papel, ya que los registradores todavía no poseen la firma electrónica avanzada, si bien se está trabajando en este tema.

Se preguntarán cuál es la utilidad entonces de este sistema. Podemos afirmar que el ahorro de costos y la mayor agilidad en los trámites es una realidad. El notario realiza la solicitud desde su oficina, y en 24 o 48 horas puede ver en la pantalla de su ordenador la información solicitada. Por tanto, puede otorgar el documento público o privado que sea necesario, según el contrato. Luego, se retirará el certificado papel a los efectos de su archivo en la carpeta del cliente.

Los avances del Gobierno Digital han llevado a la implementación de ventanillas únicas. El ejemplo que planteamos es la Ventanilla Única de Comercio Exterior. Desde el 1 de octubre de 2015, los notarios están habilitados para enviar la documentación solicitada en formato electrónico. El notario envía el certificado notarial electrónico autorizado con su firma electrónica avanzada notarial, para que el despachante de aduana correspondiente o su cliente realicen los trámites ante los organismos pertinentes. Este trámite es de gran relevancia ya que agiliza todo lo referente a exportaciones e importaciones, y ayuda a un mayor desarrollo del comercio exterior.

El Banco de la República Oriental del Uruguay, por resolución de 11 de junio de 2015, habilitó una casilla de correo para posibilitar el envío de la documentación notarial requerida vía electrónica. Es así que los notarios, utilizando el soporte notarial y su firma electrónica avanzada notarial, pueden enviar los certificados notariales electrónicos o traslados electrónicos que se les soliciten, abreviando tiempo a los clientes.

La Dirección Nacional de Catastro proporciona información de planos de los diferentes padrones baldíos o construidos, así como los valores reales de los mismos, los cuales se utilizan para la liquidación de diferentes impuestos. El notario, a través del sitio web de la misma, puede solicitar la cédula catastral y la cédula catastral informada, elementos importantes al momento de otorgar diferentes contratos sobre bienes inmuebles. En las mismas, se informa sobre el valor real del inmueble requerido, o la historia del padrón desde el punto de vista de los diferentes planos existentes. Estos son algunos ejemplos de la realidad ante la cual se encuentra el notario uruguayo a la fecha. 
A todos los sitios relacionados, el notario accede con un usuario y contraseña. Hay muchas otras oficinas que brindan servicios de interés notarial en línea, como la Intendencia de Montevideo, y de otros departamentos, Caja Notarial, Dirección General Impositiva, Banco de Previsión Social.

En cuanto a la aplicación práctica del soporte notarial y la firma electrónica avanzada notarial, si bien está vigente desde el 1 de junio de 2015, sólo dos oficinas reciben los documentos notariales electrónicos referidos por la normativa vigente: el Banco de la República Oriental del Uruguay y la Ventanilla Única de Comercio Exterior.

A dos años de la entrada en vigencia de esta importante reforma en la técnica notarial, podemos decir que los avances son lentos, tanto desde el lado de los diferentes organismos del Estado, que deben admitir la recepción de la documentación notarial electrónica, como desde el lado del gremio notarial.

Sabemos que todo cambio es resistido, pero el notariado uruguayo debe hacer los máximos esfuerzos para gestionar éste. El notariado siempre se caracterizó por asumir los cambios y adaptarse a ellos. Por eso, el gran reto que tiene en el corto y mediano plazo es profundizar en el estudio de estas nuevas herramientas tecnológicas, para así utilizarlas sin temores ni inseguridades. La seguridad jurídica y la seguridad tecnológica que el notario exige para el desempeño de su función hoy están presentes en Uruguay. Está la legislación, la seguridad jurídica y la seguridad tecnológica que nos brinda la firma electrónica avanzada.

Ante tal escenario, no tenemos más que jugar el rol para el que fuimos preparados: aplicar el derecho para otorgar seguridad jurídica preventiva y ser el fiel de la balanza entre las partes contratantes. En este mundo globalizado, donde las asimetrías entre las partes son cada vez mayores, nuestra función asesora se ve potenciada, y entendemos que es fundamental para mantener la paz social y llegar a un mayor desarrollo del comercio electrónico. Con nuestro consejo imparcial y técnico, debemos lograr que los consumidores tengan confianza a la hora de contratar a través de medios electrónicos. Sólo así se posibilitará un comercio electrónico que permita mayor desarrollo y más equidad social.

Estamos, pues, ante un escenario con nuevos actores, bases de datos, responsables de tratamiento, consumidores electrónicos, derecho de imagen y otros muchos que vendrán, ya que la velocidad en los avances de la tecnología es muy grande.

Coincidimos con Javier Wortman cuando dice: 
Toda la cantidad de nuevos hechos informáticos dará lugar a nuevas formas de recolección de evidencias electrónicas y nuevas formas de preconstituir pruebas, con un rol sumamente importante de nuestros colectivos, pero además dará lugar a nuevos mecanismos para la resolución de los conflictos que se puedan plantear en estos entornos, tales como la cibermediación, la ciberconciliación e incluso el ciberarbitraje. ${ }^{11}$

Las tecnologías nos abren nuevos nichos de mercado que debemos visualizar. Siguiendo a Julia Siri, el objeto de la función notarial es el "asesoramiento cautelar". ${ }^{12}$ El notario es el tercero imparcial en toda relación de partes, el que debe informar a las mismas de los beneficios y riesgos del negocio jurídico. Es por ello que decimos con total firmeza que hoy esta función asesora adquiere mayor relevancia. Los nuevos actores que aparecen en juego son la mayoría de las veces desconocidos para las partes contratantes, sobre todo, para el "ciudadano común”, parte débil en cualquier contratación electrónica.

Creemos que el cambio vale la pena y debemos comenzar ya. Para ello, el notariado nacional, dadas sus características, debe tener especial cuidado para que estas nuevas herramientas no profundicen la brecha que hoy ya existe, sino que posibilite a todos llegar a un ejercicio digno de nuestra profesión.

\section{Conclusiones}

El notariado uruguayo debe gestionar el profundo cambio que se le presenta en el ejercicio de su función con firmeza, sin dejar de lado principios irrenunciables de la función notarial, como el control de legalidad y el consentimiento informado.

Como gremio, debe tener metas concretas a corto y mediano plazo. Profundizar en el estudio de estos nuevos paradigmas que la tecnología nos presenta, sabiendo que no se trata de un trabajo de meses, sino que, dada la gran velocidad de cambio de la tecnología, deberá ser un estudio serio y continuo.

Comenzar a gestionar la nueva relacion con el Estado, con los colegas y con los ciudadanos. El gran desarrollo de internet y las redes sociales nos

\footnotetext{
"WoRTMAn, JAVIER, "La función notarial y la aplicación de las nuevas tecnologías. Ser o no ser digital. Esa es la cuestión", Revista de la Asociación de Escribanos del Uruguay, tomo 100. [Consulta: 23 de marzo, 2017]. Disponible en: http://biblioteca3.aeu.org.uy/digital/RAEU/100/100-1-139-155.pdf

${ }^{12}$ SirI Garcia, Julia, "Responsabilidad civil del escribano. El llamado seguro de mala praxis. Sus diferencias con el seguro de título", Revista de la Asociación de Escribanos del Uruguay, tomo 90. [Consulta: 17 de septiembre, 2017]. Disponible en: http://biblioteca3.aeu.org.uy/digital/RAEU/090/090-E-57-111.pdf
} 
exige involucrarnos con ese nuevo relacionamiento social y laboral, para seguir siendo notarios eficientes en el ejercicio de nuestra función.

Debemos participar activamente en la contratación electrónica, para seguir otorgando seguridad jurídica. El trabajo del notario en el comercio electrónico permitirá acortar la brecha entre las partes contratantes.

Asimismo, hay que promover una reforma del Reglamento Notarial en cuanto al uso de las diferentes herramientas tecnológicas que la innovación propone, inclusive, de la firma electrónica avanzada notarial. También es necesario asesorar a las autoridades competentes en la materia, para que pueda incluir los rápidos avances tecnológicos.

Debemos poner nuestra gota de agua en este gran océano globalizado, y como técnicos de derecho, debemos seguir acompañando al ciudadano y lograr sociedades más democráticas, más equitativas y más inclusivas. 


\section{Bibliografía}

“Acordada 7533 del 22 de octubre de 2004. Reglamento Notarial”, Poder Judicial de la República Oriental del Uruguay. [Consulta: 15 de febrero, 2017]. Disponible en: http://www.poderjudicial.gub.uy/images/stories/circulares/2004/100ACORDADA7533REGLAMENTONOTARIAL-TEXTOCOMPLETO.PDF

“Acordada 7831 del 4 de febrero de 2015”, Poder Judicial de la República Oriental del Uruguay. [Consulta: 15 de febrero, 2017]. Disponible en: http://www.poderjudicial.gub.uy/images/stories/circulares/2015/006-15_-_REF._ACORDADA_7831_-_Firma_electr\%C3\%B3nica_avanzada_notarial-1.pdf

“Agenda Uruguay Digital 2020: transformación con equidad”, Uruguay Digital. [Consulta: 14 de abril, 2017]. Disponible en: http://uruguaydigital.gub.uy/ wps/wcm/connect/urudigital/8f392a86-be63-4dcf-98d9-162e0933bb7f/ Agenda+Uruguay+Digital+2020+-+final+ES.PDF?MOD=AJPERESEtContentCache $=$ NONE

“Anexos a las notas a los estados contables. Nota 11". Caja Notarial. [Consulta: 15 de septiembre, 2017]. Disponible en: http://www.cajanotarial.org.uy/innovaportal/file/143/14/anexos-ec-2016.pdf

AynÈs, LAURENT, L'authenticité, París, La Documentation française, 2013.

Bouvier, Elisabeth y Pereiro Alonso, María Claudia, "El notario: participa o se involucra en la sociedad de la información”, Asociación de Escribanos del Uruguay, Montevideo, Asociación de Escribanos del Uruguay, 2012.

“Ley 18600 del 21 de setiembre de 2009”, Centro de Información Oficial Impo. [Consulta: 15 de diciembre, 2016]. Disponible en: http://www.impo.com.uy/bases/leyes-originales/18600-2009

“Ley 18996 del 7 de noviembre de 2012”, Centro de Información Oficial Impo. [Consulta: 5 de febrero, 2017]. Disponible en: http://www.impo.com.uy/bases/ leyes-originales/18996-2012

“Ley 19355 del 30 de diciembre de 2015”, Centro de Información Oficial Impo. [Consulta: 5 de febrero, 2017]. Disponible en: http://www.impo.com.uy/bases/ leyes-originales/19355-2015

Moreau, Alain, La magistratura de prevención o el notario agente de protección de los derechos del hombre, 1999.

Siri García, Julia, "Responsabilidad civil del escribano. El llamado seguro de mala praxis. Sus diferencias con el seguro de título", Revista de la Asociación de Escribanos del Uruguay, tomo 90. [Consulta: 17 de septiembre, 2017]. Disponible en: http://biblioteca3.aeu.org.uy/digital/RAEU/090/090-E-57-111.pdf

“Solicitud de papel y soporte electrónico", Caja Notarial. [Consulta: 14 de abril, 2017]. Disponible en: http://www.cajanotarial.org.uy/innovaportal/v/2280/1/innova.front/solicitud-de-papel-y-soporte-electronico.html 
"Trámites en línea", Agesic. [Consulta: 5 de mayo, 2017]. Disponible en: https:// www.agesic.gub.uy/innovaportal/v/6020/21/agesic/como-vamos.html?idPadre $=4588$

"Uruguay en cifras 2014", Instituto Nacional de Estadística. [Consulta: 15 de setiembre, 2017]. Disponible en: http://www.ine.gub.uy/documents/10181/39317/ Uruguay en cifras 2014.pdf/aac28208-4670-4e96-b8c1-b2abb93b5b13

WORTMAN, JAVIER, "La función notarial y la aplicación de las nuevas tecnologías. Ser o no ser digital. Esa es la cuestión", Revista de la Asociación de Escribanos del Uruguay, tomo 100. [Consulta: 23 de marzo, 2017]. Disponible en: http:// biblioteca3.aeu.org.uy/digital/RAEU/100/100-1-139-155.pdf 